\title{
Las reformas a la protección social en salud en México: ¿rupturas o continuidades?
}

\section{Mónica Uribe Gómez* Raquel Abrantes Pêgo ${ }^{* *}$}

\section{Resumen}

Desde la década de los ochenta comenzó un proceso de reformas al sistema de salud mexicano con el fin de promover cambios estructurales en el sector mediante una mayor presencia del sector privado. Se argumenta que la reforma fue propuesta por un grupo de actores tecno-burocráticos que han concentrado recursos de poder cruciales para modificar las reglas institucionales y que tales cambios se han llevado a cabo sin consultar ampliamente a los diversos actores interesados: gobiernos de los estados de la república, proveedores de los servicios públicos y usuarios.

\section{Abstract}

This paper examines the reform process within the Mexican health system undertaken since the eighties that led to an increasing privatization of health services. It argues that such process have been led by a small group of techno-bureaucratic actors that have changed institutional rules according to their interests without considering the interests of local governments, public services providers and public health services users.

Palabras clave: reforma de la atención de salud, protección social en salud, Seguro Popular, proceso político, México.

Key words: health care reform, public policy, Popular Insurance, political process, Mexico.

* Profesora de la División de Ciencias Sociales y Humanidades de la Universidad de Guanajuato.

** Investigadora del Centro Interamericano de Estudios de Seguridad Social.

Las autoras agradecen al profesor Mario Hernández y a su equipo de investigación de la Universidad Nacional de Colombia la posibilidad de participar en las discusiones de su proyecto sobre los procesos de reformas del sector salud en Colombia, México y Brasil. 
esde la década de los ochenta, el sistema de salud instaurado en México, como parte constitutiva del proyecto social del Estado posrevolucionario, ha pasado por distintas modificaciones que han intentado disminuir la fragmentación estructural entre institutos de seguridad social y asistencia pública en salud. Las reestructuraciones han comprendido una reforma constitucional para reconocer el derecho a la protección en salud e incluir al sector privado como parte constitutiva del sector salud; la sustitución del sistema de reparto en las pensiones por uno de cuentas individuales en el sector de la seguridad social; la introducción de mecanismos de mercado, y la incorporación de los principios de la descentralización en el manejo de los recursos y programas de salud. No obstante esas reformas, este trabajo argumenta que subsisten problemas de fragmentación, ineficiencia e inequidad en el sistema de salud mexicano.

A partir del año 2000 se hicieron nuevos intentos para reorganizar el llamado Sistema Nacional de Salud (SNS). Particularmente, se llevó a cabo la reforma a la Ley General de Salud mediante la cual se creó el Sistema de Protección en Salud (SPSS), cuyo componente operativo es el Seguro Popular (SP). Esta iniciativa surgió a partir de la propuesta de alcanzar la cobertura universal para un número delimitado de intervenciones por medio de un instrumento financiero de aseguramiento. También se propuso disminuir el gasto de bolsillo en salud con la creación de un sistema de incentivos que permitiera subsidiar la demanda en vez de la oferta como lo había hecho hasta entonces la Secretaría de Salud (SSA). Según su propio ideólogo y promotor, la inversión insuficiente en salud ante el nuevo perfil de enfermedades, junto a la creciente demanda de los ciudadanos por obtener servicios de alta calidad con protección financiera para todos, motivaron la reforma a la Ley General en Salud de 2003 que dio origen al SP (Frenk, 2012).

Este trabajo plantea que en el diseño y estrategias de implementación del SPSS, no sólo han influido la necesidad manifiesta de mejorar los servicios de salud, atender las demandas sociosanitarias o adecuarse a las tendencias internacionales del desarrollo sino también una serie de arreglos político-institucionales impulsados por un grupo de actores del sector. Se argumenta que a pesar de que esos actores lograron institucionalizar un modelo de salud, ese grupo no logró invertir las tendencias en la inequidad e ineficiencias del SNS, ni disminuir el gasto de bolsillo de los más pobres. En este sentido, interesa responder a preguntas tales como ¿cuáles han sido los enfoques y prioridades de la protección social en salud en México desde los años ochenta?, ¿qué actores han tenido 
mayor peso en las decisiones y por qué?, ¿cómo se han materializado - o no - los intereses y discursos de los diversos actores en las reformas aprobadas? En el primer apartado se exponen algunos de los puntos centrales del modelo que ha sustentado las reformas de la salud desde los años ochenta. A continuación se presentan algunos antecedentes de las transformaciones, en donde se resalta la etapa de reformas del Instituto Mexicano de Seguridad Social (IMSS) en 1996; posteriormente se analiza el proceso de creación del SPSS en 2004 y se muestran algunas de las contradicciones y dificultades de su implementación. Finalmente, se retoman algunos de los elementos discutidos para hacer una reflexión en torno a las tendencias que plantean la necesidad de una "nueva" reforma a la protección en salud en México.

\section{El modelo de salud detrás de las reformas}

Las reformas del sector de la salud en América Latina fueron parte de las políticas destinadas a "modernizar" el Estado, mismas que se desarrollaron en el marco de los ajustes estructurales promovidos por los organismos internacionales desde los años ochenta como respuesta a la crisis de la deuda externa. Desde entonces, la protección financiera se definió, en los discursos de los reformadores, como el parámetro más importante de la protección en salud. Aunque indudablemente gran parte de los sistemas de salud latinoamericanos presentaban serios problemas de fragmentación institucional, déficit de financiamiento, cobertura insuficiente y baja efectividad, en la decisión de iniciar los ajustes tuvo mayor peso la motivación por disminuir el gasto social estatal, negociar las condiciones del reembolso de la deuda con los organismos prestamistas y adaptar el sector a los nuevos parámetros del mercado, que la necesidad de dar respuesta a las necesidades sanitarias de sociedades cada vez más complejas y demandantes de mejores servicios de salud.

El interés del Banco Mundial (BM) por las reformas de las políticas de salud de la región se explicitó en el informe de 1987 Financing Health Services in Developing Countries. An Agenda for Reform, en el cual se diferenciaron los bienes públicos en salud de los privados y se propusieron cuatro estrategias para financiar la atención en contextos de austeridad: 1) instauración de co-pagos; ${ }^{1}$ 2) establecimiento de incentivos para el desarrollo de

\footnotetext{
La introducción de los co-pagos buscaba, según los reformadores, fortalecer la financiación del sistema y estimular el uso racional de los servicios de salud.
} 
seguros de salud; 3) separación de funciones, y 4) descentralización de los servicios de salud. Este enfoque se articulaba con otras iniciativas discutidas desde finales de los años setenta por instituciones como la Fundación Rockefeller, que abogaba por la atención primaria selectiva (en vez de la integral, como se había planteado en Alma Ata en 1978), y argumentaban en favor de dirigir principalmente las acciones de la salud hacia los grupos de alto riesgo. Dicha orientación prescindía por completo de una agenda de debate liderada por salubristas en torno al modelo de atención y a la necesidad de articular los temas de salud, democracia y condiciones de vida.

En el informe Invertir en salud (1993), publicado unos años después por el BM, se hizo un análisis detallado de las políticas sanitarias y de su relación con el desarrollo económico. En el capítulo dedicado a los sistemas de salud, el informe menciona la necesidad de cambiar las estructuras existentes para garantizar la equidad y la asignación adecuada de los recursos a partir de la relación costo-eficiencia y de la implementación de estrategias de "autofinanciación" (materializadas en co-pagos para los usuarios en función de su nivel de ingresos). Según las recomendaciones de dicho informe, los gobiernos sólo debían financiar un paquete reducido de servicios, de tal manera que se garantizara la asignación racional y la rentabilidad de los recursos. La idea detrás de los paquetes básicos de salud era priorizar la atención a los principales padecimientos de la población, cuya atención no representaba altos costos, pero sí tendría un impacto notable al maximizar el número de "años de vida saludable". Estos parámetros conformaron la base de la propuesta tomada posteriormente como modelo para las reformas en salud, mejor conocida en la región como "Pluralismo Estructurado para la Salud". Esta iniciativa de reformas presentada a mediados de los noventa fue promovida por el médico salubrista mexicano Julio Frenk, entonces miembro de la Fundación Mexicana para la Salud (Funsalud), y por el economista y ex ministro de salud colombiano Juan Luis Londoño, vinculado en esos años con el BM.

El modelo de "pluralismo estructurado" sostiene que para lograr la equidad y la eficiencia en los sistemas de salud deben separarse las funciones de regulación y administración del financiamiento de las de prestación de los servicios, elementos que, combinados, deberán reflejarse en la disminución de los costos y el aumento en la calidad de los servicios. Según esta propuesta, el Estado debe ser el responsable de la rectoría y modulación del sistema de salud; mientras el aseguramiento y la prestación de servicios deben estar a cargo de distintas entidades públicas y privadas que actuaran en un marco de "competencia regulada" (Londoño y Frenk, 1997). Mientras se buscaba que otros actores (usuarios y entidades territoriales) 
participaran en la financiación del sector, el Estado debía concentrarse en la dirección y en la regulación del sistema de salud. Esta perspectiva, sin embargo, no incluye otros requerimientos sociales, como que dicha regulación también evite la concentración de recursos y garantice la protección del interés general sin menospreciar la participación de los actores colectivos en la toma de decisiones (Almeida, 2005).

El diseño de estas reformas se basó en los análisis de Alain Enthoven (1980), mismos que, según el autor, no se aplicaban más que al sistema de salud norteamericano. Según un analista, "[la propuesta de Enthoven] se centró en la lógica del mercado de aseguramiento, de manera que los competidores no estuvieran estimulados por el precio de la póliza sino por el número de afiliados, una mayor eficiencia en la operación y la libre elección" (Hernández, 2008: 78). Asimismo, el sistema de salud debería funcionar bajo estándares de costo-beneficio y establecer normas para regular los mecanismos de afiliación y financiamiento.

Posteriormente, el modelo de "mercado regulado" se complementó con el Manejo del Riesgo Social (MRS), también promovido por el BM desde el año 2000, el cual se presentó como una alternativa para reorientar la protección social. El modelo del MRS planteaba que uno de los problemas de la protección social en el pasado había sido la orientación hacia medidas públicas de respuesta que mostraron sólo un éxito moderado. Por lo tanto, las acciones del Estado debían focalizarse en subsidios a la demanda y concentrarse en los usuarios definidos como pobres, es decir, los usuarios más vulnerables a los riesgos naturales y sociales sin acceso a las dinámicas del mercado laboral (Holzmann y Jorgensen, 2000).

De manera general, los aspectos mencionados resumen la perspectiva en la que se fundamentaron las reformas realizadas al sistema de salud mexicano desde los años ochenta.

\section{Las reformas iniciales: entre la segmentación originaria y el pluralismo estructurado}

A la vez que la reforma constitucional de 1983 declaró el derecho a la protección en salud para todos los mexicanos en un contexto político en el que el Partido Revolucionario Institucional (PRI) todavía conservaba la mayoría en la cámara, también, paradójicamente, descartó la idea de gratuidad en los servicios aun para los más pobres. Esta reforma además abrió la posibilidad de participación a la competencia entre los prestadores de servicios públicos y privados. Dicha modificación constitucional 
estableció que la Ley General de Salud - promulgada en 1984 - definiría las bases y modalidades para el acceso a los servicios de salud y establecería la concurrencia entre la federación y las entidades federativas en materia de salubridad general (artículo 4). ${ }^{2}$ Los cambios legislativos sirvieron como base para fortalecer la descentralización de los servicios de salud para la población no asegurada. ${ }^{3}$ Aun con la reforma constitucional no se habían resuelto los problemas estructurales de segmentación, de falta de financiamiento y de cobertura insuficiente del sistema de salud. Por lo tanto, la necesidad de reestructurar el sector adquirió un lugar importante en las agendas públicas. Desde principios de los noventa, el Centro de Desarrollo Estratégico para la Seguridad Social (CEDESS), ente creado por la dirección del IMSS durante el gobierno de Carlos Salinas de Gortari (1988-1994), había abogado por la privatización del sector de la salud. Sin embargo, dichas recomendaciones no fueron acogidas por el presidente Salinas quien, en aquel momento, prefirió no dar continuidad a la descentralización y promover, a través del Programa Nacional de Solidaridad, una base de legitimidad popular alejada de los viejos esquemas corporativos, al tiempo que crecía la expectativa de crecimiento de la privatización del sistema de pensiones ${ }^{4}$ (Valencia et al., 2011; González-Rossetti y Mogollón, 2002, Abrantes, 2010).

Durante la presidencia de Ernesto Zedillo (1994-2000), se retomó el interés por reestructurar el sector salud y se creó el Programa de Reforma del Sector Salud que adoptó el modelo de pluralismo estructurado. Como parte de esta estrategia, entre otras medidas, se propuso la reorganización de la atención y del sistema de pensiones del IMSS, la continuación de las medidas de descentralización iniciadas en 1984 y la elaboración de paquetes de atención en el ámbito de la Ss. Como parte de tales cambios, a mediados de los años noventa, el entonces director del IMSS Genaro Borrego Estrada presentó el documento Diagnóstico en el que mostraba el estado de las finanzas del IMSS en una grave crisis y la ingente necesidad de intervenir para evitar su desaparición. El documento exponía que si no se tomaban las medidas suficientes, no se lograría formar un fondo suficiente para la pensión de un asegurado y para financiar los servicios de salud, ni

2 Esta reforma fue adoptada sin mayores problemas, puesto que el pri, todavía en el poder, controlaba los votos con 299 diputados (75\%) contra 51 del pan (13\%) y 100\% de los senadores.

3 Para ampliar la perspectiva sobre el proceso de descentralización puede consultarse Homedes y Ugalde (2006; 2011).

4 Esa iniciativa fue vetada por el líder sindical Fidel Velázquez, y como consecuencia la reforma se redujo a la instauración del Sistema de Ahorro para el Retiro (SAR) (Brachet-Márquez, 2007) lo que provocó la salida del entonces director del IMSS, Ricardo García Sainz (Abrantes, 2010). 
siquiera cotizando durante el tiempo máximo permitido de 40 años (IMSS, 1995). Entonces, el gobierno planteó, la necesidad de privatizar el sistema de pensiones, de garantizar la reversión de cuotas ${ }^{5}$ para las aseguradoras que atendieran a los trabajadores afiliados al IMSS y de separar los diferentes beneficios de sus fuentes de financiamiento con el fin de asegurar su viabilidad financiera.

Según el Sindicato Nacional de Trabajadores del Seguro Social (SNTSS), las medidas sugeridas eran parciales, sesgadas y tendenciosas. En la opinión de los miembros de dicho sindicato, se había responsabilizado al IMSS de la crisis, al hacer caso omiso del origen de su déficit en la política económica en marcha que significaba merma en el empleo, base del financimiento de la institución. En los debates de este periodo, sobresalió la posición de un grupo de salubristas de medicina social, quienes junto con el entonces director del IMSS Ricardo García Sainz, y en alianza con el Partido de la Revolución Democrática (PRD), cuestionaron las bases técnicas del Diagnóstico y argumentaron que existía una agenda oculta que buscaba manipular la información en tanto se mezclaban problemas reales con otros no demostrados (Abrantes, 2010).

El SNTSS, para entonces, contaba con 350 mil afiliados, y era de conocimiento público el vínculo de sus líderes con el PRI. Una muestra de la capacidad de movilización de este grupo fue la organización del Foro "Beneficios, costos y financiamiento de la seguridad social" en julio de 1995, que contó con la asistencia de más de 500 representantes de organizaciones sindicales, patronales y civiles. En el evento, el sindicato manifestó la intención de modificar el voto corporativo-sindical en favor del PRD y en contra del PRI en las siguientes elecciones (Abrantes, 2010; Ulloa Padilla, 1996). Entre los temas que generaron mayor oposición a la reforma estaban la reversión de cuotas y la subrogación de servicios, cuestiones que encaminaban la posibilidad de abrir la competencia en la prestación de los servicios de salud del IMSS al sector privado (Brachet-Márquez, 2007; Sáenz, 1996).

El primer proyecto de reforma presentado por el Ejecutivo y aprobado en diciembre de 1995 con el voto del PRI no contó con el apoyo del SNTSS que argumentaba que la iniciativa gubernamental era parte de las estrategias reformistas del BM para privatizar los servicios de salud y materializar la separación de las funciones de financiamiento y de prestación de los servicios. En el debate parlamentario que siguió, la oposición (el SNTSS y el

5 La reversión de cuotas y la subrogación de servicios permiten transferir las cuotas obrero-patronales a las aseguradoras privadas para que estas empresas provean servicios médicos a los derechohabientes del IMSS. 
PRD) consiguió la modificación de más de 60 artículos que representaban la pérdida de derechos adquiridos por los trabajadores. La propuesta definitiva no fue aprobada con todos sus cambios sino hasta finales de 1996, y entró en vigor a mediados de 1997 (Boltvinik, 1998; Brachet-Márquez, 2007; Abrantes, 2006).

Aunque muchos de los cambios inicialmente contemplados por los reformadores fueron ratificados, otros se derogaron, como los artículos 89 y 213 relacionados con la reversión de cuotas y con la posibilidad de convenios con empresas privadas para la prestación de servicios de salud para los derechohabientes del IMSS. Esto significaba que el modelo integrado con el que habían sido creadas las instituciones de seguridad social se mantendría - en parte - , a la vez que la reforma significaba un fracaso para quienes pretendían introducir la separación de funciones entre financiadores y prestadores de servicios en la institución (Laurell, 2010: 143). Sin embargo, aunque se evitó la privatización de la salud, esta reforma encauzó finalmente las pensiones a la iniciativa privada, lo que significaba que el único rubro redituable del presupuesto institucional quedaba a disposición de la competencia de mercado, ${ }^{6}$ a la vez que dejaba al IMSS con menos capacidad para financiar el seguro de enfermedad y maternidad tradicionalmente deficitario (Brachet-Márquez, 2007; IMSS, 1996).

En el ámbito de la Secretaría de Salud, Funsalud trató de que algunas de las sugerencias que había realizado para la reforma fueran incluidas (Funsalud, 1994), específicamente, separar las funciones de la SSA, avanzar en la descentralización y generar un paquete básico de servicios para la llamada "población abierta". Esas iniciativas eran parte del Programa de Reforma del Sector Salud financiado con recursos del BM (López y Blanco, 2007). Finalmente, en 1995 se estableció el primer paquete de servicios y tratamientos que incluía 13 intervenciones como parte de las acciones básicas de salud y se avanzó en la descentralización.

Los años noventa terminaron con un sistema de protección en salud que seguía segmentado entre distintas instituciones que funcionaban de manera independiente. Aunque en la SSA se incorporaron de manera incipiente algunas estrategias del modelo de "pluralismo estructurado" con la separación - relativa - de las funciones de financiamiento y de prestación de los servicios, y con la instauración de un primer paquete de intervenciones, en otras instancias, como en el IMSS, se mantenía tanto la integración

6 Con esta reforma se incrementó el tiempo de cotización de 500 a 1200 semanas, se crearon cuentas de capitalización individual, y se delegó la administración del fondo pensional a instancias privadas conocidas como Afores. 
de las funciones como la amplia cobertura en las intervenciones médicas, hospitalarias y farmacéuticas de sus asegurados y familiares, pero con una capacidad financiera disminuida como resultado de la reforma.

\section{Creación del Sistema de Protección Social en Salud (SPSS)}

El SNS arribó al siglo XXI con claros rezagos pese a las reestructuraciones previamente introducidas. Julio Frenk y sus colaboradores continuaban presionando, ahora desde la Organización Mundial de la Salud (OMS), para alterar las bases fundacionales del sistema de protección social mexicano. En un polémico informe publicado en el año 2000 sobre los sistemas de salud en el mundo, en una lista de 191 países, México apareció en el lugar 51 en materia de desempeño, mientras que en equidad financiera ocupó el lugar 144. Frenk, quien para entonces estaba encargado del área de investigación e información para las políticas en la OMS, fue uno de los redactores de este documento en el que expuso la importancia de instaurar seguros de salud y estrategias de co-pago que, según él, eran la mejor opción para proteger a las personas de la depauperización ocasionada por el llamado gasto catastrófico y empobrecedor en salud. ${ }^{7}$ Ese documento fue criticado y cuestionado por algunos expertos en el tema (Navarro, 2001: 34; González, 2001), que calificaron de parcial la información divulgada debido a que centraba los problemas de los sistemas de salud en el componente financiero, lo cual no necesariamente coincidía con la percepción de los usuarios sobre la efectividad y el acceso a los servicios.

Durante esos años también se presentaron cambios políticos que se observaban como rupturas en las formas de negociar, asignar los recursos y promover acciones en función de las políticas públicas establecidas. El PRI había perdido la presidencia y la mayoría en el Congreso, y Vicente Fox, candidato del Partido de Acción Nacional (PAN), había sido elegido presidente. Aunque en los inicios de aquel gobierno fueron frecuentes las críticas a la lógica del "pacto corporativo", 8 instaurado durante el priísmo, en la práctica los mecanismos institucionales para regular el ejercicio del poder

7 Para ampliar el contenido de estas propuestas puede consultarse el comunicado de prensa divulgado por la OMS en junio de 2000 sobre la evaluación de los sistemas de salud en el mundo, disponible en: https://apps.who.int/whr/2000/pdf-sp/Pressrelease-sp.pdf

8 Se entiende la expresión "pacto corporativo" en los términos mencionados por Brachet-Márquez (1996), quien plantea que el México posrevolucionario se caracterizó por la consolidación de un tipo de corporativismo de Estado que dividió a la población en los sectores obrero, campesino y popular, de los cuales dependía la estabilidad del régimen político. Cada sector se incorpo- 
habían sufrido relativamente pocas modificaciones, como lo demostró el proceso político que se siguió para crear el SPSS.

El Plan Nacional de Salud (2001-2006), conocido como La democratización de la salud en México, ${ }^{9}$ incorporó los aspectos más relevantes del modelo de reforma que prosperaba desde los años ochenta. Este documento contenía la propuesta de creación del Sistema de Protección Social en Salud (SPSS), cuyo componente operativo sería el Seguro Popular (SP). Dicha reestructuración se formalizó a partir de $2004^{10}$ (con la meta de que para $2010 \mathrm{cu}$ briría a la totalidad de la población). Para financiar el SP, se estableció una modalidad tripartita con una aportación federal de $80 \%$, es decir, los porcentajes restantes procederían de las contribuciones estatales y de las aportaciones de los hogares, y se exentaría de pago a las familias inicialmente pertenecientes a los deciles de ingresos I y II, y a partir de 2010 también a los deciles III y IV.11 Con esa reestructuración, los niveles estatales y municipales recibieron la responsabilidad de garantizar tanto los servicios de salud pública (v. gr. la vigilancia epidemiológica, la protección ambiental y la protección contra riesgos sanitarios) como la atención individual. Esta última contemplaba servicios esenciales(de primer y segundo nivel) y de alto costo. Los primeros forman parte del Catálogo de Servicios Esenciales de Salud (CAUSES), mientras que los de alto costo se financian con el Fondo de Protección contra Gastos Catastróficos (FPCGC). ${ }^{12}$

ró al partido oficial según condiciones particulares y con un conjunto propio de derechos a las prestaciones de la legislación social.

9 El Plan Nacional de Salud 2001-2006 estableció las directrices para el sector durante el periodo en que Frenk fue secretario de Salud. Este documento plantea que la democratización de la salud significa la creación de las condiciones para que se dé la universalización del acceso a la salud, la estimulación de la participación ciudadana y la garantía de calidad en los servicios (Secretaría de Salud, 2001). El acceso universal aludido se restringe a un paquete de intervenciones acotadas y de mucho menor alcance a las que corresponden a los beneficiarios de las instituciones de la seguridad social, lo que constituye de partida un acceso diferenciado e inequitativo del derecho a la salud.

10 El SPS tenía como antecedente un plan piloto implementado desde 2001 en cinco estados de la República: Aguascalientes, Campeche, Colima, Jalisco y Tabasco.

11 En 2012 el gobierno federal aportó una cuota social, por cada persona afiliada, de 880.19 pesos. Las aportaciones solidarias de los estados equivalen a la mitad de la cuota social y la cuota familiar de los beneficiarios del nivel $\mathrm{V}$ en adelante es pagada anualmente y determinada por el nivel de ingreso. (Grupo de Trabajo Funsalud, 2013: 8.)

12 En un principio, el paquete de servicios fue conocido como Catálogo de Servicios Médicos (CABEME), el cual contemplaba 78 intervenciones. A partir de 2004 el paquete empezó a ser conocido como Catálogo Esencial de Servicios (CASES) y ampliado a 91 intervenciones. Posteriormente, en 2005, se aumentarían a 155 intervenciones. Por último, en 2006, el paquete empezó a ser conocido como Catálogo Universal de Servicios (CAUSES), el cual, en 2012, se había ampliado a 284 intervenciones de primero y segundo nivel. Por su parte, el Fondo de Protección contra Gastos Catastróficos (FPCGC) cubre 17 padecimientos de alto costo como el cáncer cérvico-uterino 
Julio Frenk fue el artífice y promotor de esta reforma cuando estuvo a cargo de la SSA entre 2000 y 2006. En distintos espacios académicos, políticos y medios de comunicación, el entonces secretario defendió personalmente las bondades de las modificaciones al sistema de salud. La discusión y gestión de esta iniciativa estuvo principalmente en manos del secretario de Salud y su grupo técnico de apoyo. En un principio, los reformadores contemplaron la posibilidad de que se pudieran conformar las distintas instituciones mediante la integración de un sistema complementario de servicios conformado por los prestadores de la SSA, el IMSS y el Instituto de Seguridad Social y Servicios Sociales de los Trabajadores del Estado (ISSSTE). Sin embargo, según un testimonio del mismo Frenk (2005), después de realizar algunos análisis con el director de dicha institución, se descartó esta idea inicial, debido a las dificultades para llegar a un acuerdo sobre la dirección que debían seguir las transformaciones.

Desde un principio, el SNTSS rechazó el SP con el argumento de que dicho programa, lejos de resolver los problemas de los más pobres, debilitaba a las instituciones de seguridad social ya consolidadas, al perder autonomía y verse obligadas a competir con el sector privado en condiciones desiguales. Las diferentes perspectivas sobre la seguridad social y los conflictos laborales que se vislumbraban con estas modificaciones llevaron a abandonar, en 2002, la meta de integrar al sistema de salud. Los cambios propuestos también enfrentaron la resistencia de los representantes de los estados, quienes, bajo el nuevo esquema, debían empezar a contribuir en la financiación del programa. En respuesta, los reformadores plantearon la posibilidad de eliminar este aporte, pero cuando el proyecto se presentó ante el Congreso, se incluyeron las "aportaciones solidarias", las cuales establecían el monto que aportarían las entidades federativas (Lakin, 2010: 320-322), cuyos presupuestos de salud se asignarían en proporción al número de personas afiliadas al SP.

Funsalud, por su parte, fue un actor decisivo en el diseño de las reformas. Esta fundación privada reunía - y lo sigue haciendo - a personajes y posturas institucionales que respaldan el modelo que fundamentó la creación del SPSS. Los estudios y recomendaciones de los especialistas de Funsalud han sido el principal referente para documentar el carácter "científico" de las evidencias que han justificado las modificaciones del sistema

y de mama, el VIH-sida, los cuidados intensivos neonatales, las cataratas, el cáncer de niños y adolescentes, el trasplante de médula ósea y los trastornos quirúrgicos, congénitos y adquiridos, el cáncer de próstata y el trasplante de riñón. 
de salud desde los años ochenta. ${ }^{13}$ No obstante, estos especialistas poco se han preocupado por demostrar la mayor eficiencia de los sistemas de salud privados o el impacto del co-pago en el uso racional de los servicios por parte de los usuarios.

Para llevar a cabo estas modificaciones, el proceso legislativo se caracterizó por la actuación del partido en el poder (PAN) como bloque de apoyo a las propuestas del secretario de Salud. En esa oportunidad, aunque el PRI también respaldó el contenido general del proyecto, buscó que se incluyeran límites al crecimiento del sector privado. Sin embargo, según Laurell (2009), a pesar de estas demandas iniciales, una vez aprobada la ley, el Ejecutivo expidió un reglamento para volver a incluir al sector privado sin que el PRI impugnara dicha medida. ${ }^{14}$ Por su parte, como había sucedido en el proceso de reformas del IMSS, el PRD volvió a ser el opositor más fuerte de la iniciativa del Ejecutivo. La posición del partido de izquierda en los debates iniciales - siendo algunos de sus miembros integrantes de la Comisión de Seguridad Social - era que las reestructuraciones no apuntaban a resolver verdaderamente los problemas estructurales del sistema de salud. Además, consideraba que esta reforma abría la puerta a la privatización de la seguridad social. Sin embargo, en ese momento, el equipo reformador había conseguido relegar a la oposición parlamentaria y obtener el apoyo de la mayoría en el Congreso, lo que implicaba que la correlación de fuerzas estuviera claramente en favor de la aprobación de la reforma.

Al privilegiar el ámbito parlamentario en las negociaciones, los proveedores de servicios de la SSA tampoco fueron incluidos en las discusiones de la reforma, pese a que la extensión de la cobertura planeada afectaría directamente sus cargas y condiciones de trabajo. Los equipos técnicos prefirieron minimizar la participación democrática de los actores del sistema en la toma de dicisiones, sin embargo, esto no necesariamente aseguró la factibilidad política al momento de la implementación de las nuevas políticas.

La oposición a esta reforma estuvo encabezada por el partido de izquierda, PRD, el cual, a pesar de haber perdido fuerza en el espacio parlamentario, mantuvo su postura crítica frente a la propuesta del Ejecutivo. Una de

13 Un ejemplo de los análisis divulgados por la Funsalud puede encontrarse en publicaciones de la misma Funsalud como Economía y salud en México: propuestas para el avance del sistema de salud en México (1996).

14 Según Tamez y Eibenschutz (2008), la principal razón esgrimida en favor de incentivar la participación privada en la prestación de servicios de salud fue la saturación de los servicios del IMSS. 
las muestras de resistencia al modelo de salud oficial se evidenció cuando el gobierno de Andrés Manuel López Obrador se negó a implementar el SP en el Distrito Federal. Desde 2001, ese gobierno instauró el Programa de Servicios Médicos y Medicamentos Gratuitos, y operó en el contexto de fortalecimiento de los prestadores públicos, lo cual contradecía claramente la lógica de los paquetes básicos de aseguramiento impulsados por el SP, y obstaculizaba la inserción del sector privado en la prestación de los servicios de salud. Cristina Laurell, entonces secretaria de Salud del D.F., fue de las pocas en oponerse al SP en el Consejo de Salubridad. En la postura del PRD también influían otros aspectos como la posibilidad de perder la autonomía en el manejo de los servicios de salud en la ciudad de México donde este partido concentra su mayor fortaleza política (Lakin, 2010). La implementación de una política alternativa al SP con recursos financieros locales confrontaba las bases del modelo de reformas promovido por el Ejecutivo.

Estos dos modelos de salud (el del SP y el del Distrito Federal) representaban dos alternativas estructurales. La perspectiva oficial buscaba la incorporación de parámetros de costo eficiencia y la introducción de la competencia de mercado en el manejo de los recursos y la prestación de los servicios, mientras el gobierno del D.F. proponía la gratuidad de los servicios y el fortalecimiento del liderazgo del Estado en el manejo del sector. ${ }^{15}$ No fue sino hasta 2005 cuando el gobierno del D.F. aceptó solicitar su inclusión en el SP, a pesar de las diferencias que manifestaba tener con el modelo de salud que dicho programa representaba. Este hecho tuvo como marco la contienda electoral por la presidencia (2006-2012), en la que el entonces jefe de gobierno de la ciudad de México, Andrés Manuel López Obrador, participó como candidato.

A los críticos de la reforma se sumaron otras voces más pragmáticas como las del SNTSS que planteaban que medidas como el SP, en el fondo, sólo buscaban privatizar al sector. Sin embargo, el papel de los sindicatos de la seguridad social en estas discusiones fue marginal. El programa del SP no suponía ninguna consecuencia para los trabajadores que este sindicato representaba; por lo tanto, no ameritaba mayores desgastes para este gremio que no solía movilizarse por causas que no incluyeran sus intere-

15 No debe olvidarse que desde los años ochenta la doctora Laurell, al igual que el doctor Frenk, ambos críticos de los proyectos tradicionales de salud pública, se habían distanciado ideológicamente cuando se iniciaron los cambios neoliberales en el sector, que contribuyeron a polarizar a los actores del sector. Para entonces, los dos ocupaban cargos directivos en las secretarías de Salud federal y distrital, respectivamente, y desde ahí buscaron materializar los modelos de salud (opuestos) que cada uno defendía. 
ses corporativos. De alguna manera, esto ilustra, una vez más, que la mayoría de las iniciativas reformistas son acuerdos negociados que permiten que ocurra el cambio dentro de un contexto que favorece una postura de cierta tolerancia hacia las reformas por parte de los grupos de oposición a cambio de que se mantengan los privilegios conseguidos con anterioridad (Grindle, 2003). ${ }^{16}$

Irónicamente, la Secretaría de Hacienda fue otro de los actores que trató de obstaculizar la reforma al cuestionar su viabilidad financiera. Esta instancia buscó agregar un artículo que condicionara el proyecto a una reforma fiscal más amplia, aspecto que representaba un claro inconveniente para la creación del SPSS, debido a que ésta no era una iniciativa que tuviera muchas posibilidades políticas de lograrse en el corto plazo. Estos desacuerdos requirieron el incremento de las acciones de lobby de los reformadores, quienes ante las negativas de Hacienda buscaron el apoyo de la comisión financiera del Senado que trató de mediar los desacuerdos con el fin de lograr la aprobación de la reforma. Para dar vía libre a las transformaciones, se acordó que tanto el aumento de la afiliación como del presupuesto se harían de manera gradual (Lakin, 2010). Finalmente, en la primavera de 2003, después de poco más de dos años de discusión de la propuesta de reforma, se aprobó la creación del SPSS con 94\% del voto en favor en el Senado y 73\% en la Cámara de Diputados (Ortiz, 2006; Frenk, 2011).

Por otro lado, el interés de los organismos internacionales por financiar las reformas de los sistemas de salud ha sido ampliamente documentado (Infante, Mata y Acuña, 2000; Hernández, 2003; Brachet-Máquez, 2007, 2010; Banco Mundial, 2001, 2010). Desde hace dos décadas, el BM ha apoyado a México en el desarrollo del sector salud; las medidas son parte de lo que este organismo llama un paquete de cooperación técnica para mejorar el desempeño de su sistema de salud (Banco Mundial, 2010). Sin embargo, cabe anotar que contrariamente a la tesis de que estas instancias impusieron una serie de medidas, puede plantearse una adhesión político-ideológica de actores claves nacionales que en distintos momentos han sido parte de estos organismos (BM, OMS, BID) y/o han buscado desde posiciones estrategicas materializar, legitimar y defender los principios de estos organismos en materia de reformas del sector salud.

Aunque la estrategia de los reformadores de marginar a los opositores y negociar con los sindicatos, con base en el viejo estilo corporativo, apresuró

16 Éste había sido el caso en la reforma del IMSS, donde el SNTSS dejó de oponerse a la reforma en el momento en que su pensión, superior a 100\% del salario, se mantuvo en los acuerdos finales; esta cláusula se rescindió en 2005 (Brachet-Márquez, 2007). 
la formulación de un importante número de decretos y el establecimiento de las bases para el funcionamiento del SPSS en un tiempo relativamente corto, desconoció los efectos que podría tener para la implementación mantener la discusión de los cambios dentro de un grupo reducido de técnicos y especialistas que no necesariamente eran representativos de los diversos intereses de los actores del sector.

\section{La implementación de la reforma: más que un asunto técnico}

En los hechos, el sP quedó como un programa que reorganizaba los servicios de salud para la población que no contaba con otro tipo de seguridad, mediante una estructura paralela a la de los servicios ofrecidos por los estados - pero en los mismos centros de salud. Durante el gobierno de Felipe Calderón (2006-2012), el programa no sólo se mantuvo sino que añadió nuevos componentes como el seguro médico para los niños nacidos a partir de 2006 y la estrategia "Embarazo Saludable" (que busca asegurar la atención médica durante el embarazo y el parto), aspectos que equivalían a otro tipo más de segmentación y a la generación de nuevos problemas de inequidad en salud (Valencia, 2010).

La separación de funciones buscó hacerse efectiva con la creación del Consejo Nacional de Protección Social en Salud (CNPSS) que se constituyó como órgano consultivo del SPSS y con la asignación de la rectoría a la Secretaría de Salud Federal, la cual de facto había restringido su labor de regulación al subsector público de la salud, dado que la seguridad social seguía - y sigue - funcionando como un ente independiente del SP. El financiamiento se estructuró desde la lógica de los subsidios a la demanda a través del SP, mientras que se trató de introducir la competencia entre prestadores públicos y privados. Aunque en algunos estados se han venido haciendo subrogaciones de servicios (como en Jalisco donde se subrogan servicios a hospitales privados, y en Chiapas donde se subrogan al IMSS), la red de prestadores de servicios sigue estando integrada principalmente por los centros de salud y hospitales de los servicios estatales de salud. Sin embargo, la presencia privada se ha extendido en otros aspectos como la contratación de farmacias privadas para la dotación de medicamentos y en el suministro de servicios de lavandería y vigilancia.

El inicio de la operación de los servicios, por otro lado, también ha tenido inconvenientes, pues en esta fase se manifestó nuevamente el desacuerdo de los estados respecto de la aportación de las cuotas solidarias para cofinanciar el SP. Producto de estas inconformidades y de la compleja 
relación entre la federación y los estados, las contribuciones territoriales no se han materializado de la forma que fueron proyectadas en la ley. Según una nota publicada en el periódico El Universal el lunes 12 de abril de 2010, 24 entidades contaban con Regímenes Estatales de Protección Social en Salud (REPSS) dependientes de las secretarías de salud locales, por lo que no tenían capacidad técnica ni autonomía de gestión para manejar los recursos (Gómez, 2010). En esta etapa, los profesionales de la salud criticaron nuevamente que el aumento del presupuesto del sector no se había materializado ni en mejores condiciones de infraestructura ni en recursos humanos del sector (como lo había prometido la SSA durante la negociación de la reforma), lo que les dificultaba cumplir con la demanda creciente de servicios (Lakin, 2010).

Pese a que el SPSS sigue intentando adecuar sus estrategias de implementación a las condiciones actuales (adaptación de la infraestructura hospitalaria, provisión de medicamentos y de recursos humanos, etc.), y a que todavía no se cuenta con estudios de caso suficientes sobre el impacto en los diferentes estados, existen algunos elementos que ya han mostrado las limitaciones de este sistema de aseguramiento. En el caso de los recursos, pese que "el gasto total en salud como porcentaje del PIB pasó de 5.1\% en 2000 a 6.3\% en 2010" (Knaul y colaboradores, 2012: 8), no es posible afirmar que este incremento en la asignación de los recursos se haya reflejado en mejoras en los servicios de salud. Sobre este punto, el Centro de Análisis e Investigación Fundar, A.C. difundió un análisis sobre los resultados de una auditoría realizada por la federación de los estados de la República en 2010, con objeto de verificar el uso de los recursos destinados al SP. Dicho informe mostró irregularidades en una cuarta parte del presupuesto auditado en aspectos como documentación comprobatoria del gasto, adquisición de medicamentos y transferencia de los recursos de la salud a otras cuentas que tienen un impacto directo en la operación de los servicios públicos. Sobresalen casos como los del Estado de México con 69\% de irregularidades en el presupuesto; Veracruz, 57\%, y Sinaloa, 50\% (Lavielle, 2012).

Este aumento de los recursos tampoco ha logrado un impacto notorio en las condiciones de salud de los grupos tradicionalmente excluidos (los indígenas) o en la redistribución equitativa de los presupuestos entre los estados. Según un informe de la misma Funsalud intitulado "La salud en México 2006-2012", los estados del norte, generalmente entre los más prósperos del país, recibieron más recursos públicos que los del sur; esto se ejemplificó en el gasto per cápita en salud de la población no asegurada de Baja California Sur que fue de 2,128 pesos en 2005, mientras que en Guerrero fue de 773 pesos en el mismo año (Funsalud, 2006: 26). El mismo 
documento también señala que las poblaciones indígenas generalmente presentan los indicadores más bajos del país de cobertura y de uso de servicios. Sobre este último aspecto es pertinente mencionar que aunque las deficiencias en la salud indígena han sido un punto de interés reiterado en los informes de varias instancias, este problema no ha tenido prioridad en las políticas y recomendaciones de los equipos técnicos encargados de las reformas. ${ }^{17}$

La disminución en el gasto de bolsillo en salud, otra de las prioridades de la reforma, tampoco se ha materializado como se esperaba; El Grupo de Trabajo Funsalud (2013: 21) encontró que "antes de la reforma éste era de 52\%, y para 2010 sólo había descendido tres puntos". Lo que indicaría que esta meta, una de las más importantes del programa, sólo se ha logrado parcialmente. El aumento de la cobertura, otro punto central en los discursos de los reformadores, aparentemente fue más exitoso. Las cifras recientemente divulgadas por los promotores de las reformas muestran que en 2012 el programa había alcanzado la cobertura universal, al asegurar a 52 millones de mexicanos que no contaban con seguridad social (Knaul y colaboradores, 2012). ${ }^{18}$ Sin embargo, estas cifras varían de una fuente a otra. Según datos de la Encuesta Nacional y Nutrición (ENSANUT), en 2012 México tenía entre 21\% y 25\% de la población sin protección en salud. Además de otros problemas como la doble cobertura en el aseguramiento. También resaltan otros problemas, por ejemplo: la doble cobertura en el aseguramiento. Según un informe de la Auditoría Superior de la Federación, en 2010 más de 300 mil personas afiliadas al SP también eran derechohabientes del IMSS (80\%) o del ISSSTE (15\%).

Finalmente, cabe mencionar que aunque el SP había previsto mecanismos de cofinanciación para una proporción de sus afiliados, en la realidad esto sólo se ha logrado a medias debido a que el mayor número de afiliados (68\%) está concentrado en los deciles I, II, III y IV de ingreso quienes están exentos de pago (véase gráfica 1). Este dato, por un lado, muestra que son las personas más pobres quienes hacen uso del programa, y, por otro, que las estrategias para estimular el co-pago de los usuarios no han funcionado en los términos que estipula la ley. Esta extensión de la gratuidad no indica fortaleza financiera en el programa, el cual sigue mostran-

17 En esta tasa inferior de cobertura influye también la desigual distribución de los centros de salud en las zonas indígenas donde la población no cuenta con - o no puede pagar - los medios de transporte hacia los centros de salud concentrados en las zonas urbanas.

18 La mayoría de los autores que presentan tanto los balances del SP (Knaul y colaboradores, 2012) como las recientes propuestas de reforma al sector salud (Grupo de Trabajo Funsalud, 2013) han sido cercanos a Frenk desde la etapa del diseño de la reforma. 
do problemas de viabilidad financiera según los informes de los mismos reformadores (Grupo de Trabajo Funsalud, 2013:21) sino dificultades para fomentar entre los usuarios mexicanos la cultura del pago por los servicios de salud (Lakin, 2010).

\section{Gráfica 1. Distribución de la población afiliada al SP} y cuota anual familiar por decil de categorización socioeconómica

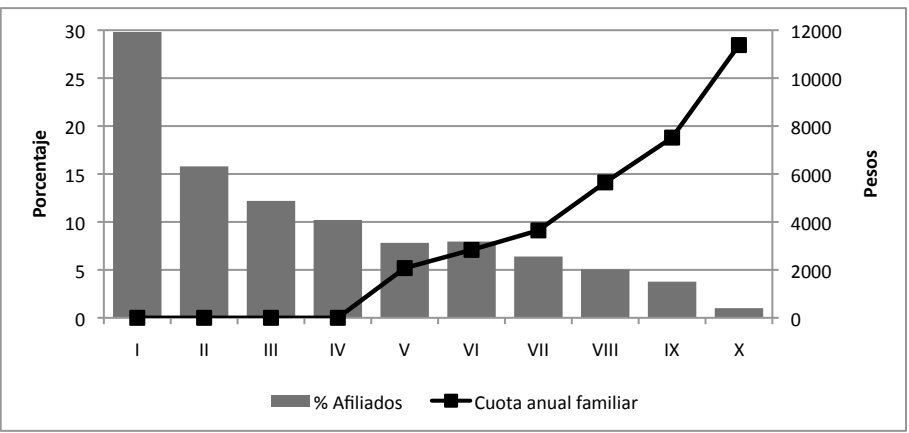

Fuente: ENSANUT, 2012

Otro punto relevante en la implementación del SP es el contenido de los paquetes básicos de salud notariamente restringido si se comparan la cobertura ofrecida por las instituciones de seguridad social, lo que afecta negativamente la equidad (Durán, 2010). Aunque las enfermedades cardiovasculares son la primera causa de muerte en el país, el paquete de intervenciones aprobado en el FPGC para los afiliados al SP sólo incluye lo relacionado con malformaciones cardiacas congénitas. En caso de infartos, sólo cubre a menores de 60 años, y deja fuera a $80 \%$ de las personas con este padecimiento (Laurell, 2012b). Por su parte, el FPGC para gastos catastróficos, excluye otros padecimientos muy costosos como la insuficiencia renal crónica, ${ }^{19}$ las transfusiones sanguíneas y las lesiones por arma de fuego. Estas exclusiones constituyen una barrera económica - y también social que afecta directamente al gasto de bolsillo en salud para las familias más pobres (Laurell, 2005).

El programa "Embarazo Saludable" está dirigido a proteger a las mujeres embarazadas con el fin de disminuir la mortalidad por causas prevenibles relacionadas con el embarazo y el parto. A pesar de la importancia de esta

19 Esta enfermedad ha presentado un crecimiento acelerado entre la población (a un ritmo aproximado de $11 \%$ anual) según reportes de la propia SS. 
iniciativa, se han detectado otros factores que explican que la mortalidad materna siga siendo un problema de salud pública en el país. Según una declaración de la Coalición por la Salud de las Mujeres, apoyada en datos del Observatorio de la Mortalidad Materna en México, para el año 2010, más de $91 \%$ de las mujeres que fallecieron por causas de muerte materna llegaron a establecimientos hospitalarios donde recibieron algún tipo de atención. Sin embargo, los servicios de emergencias obstétricas, y en general, los servicios relacionados con el embarazo y parto presentan, en muchos estados, deficiencias en la dotación hospitalaria y problemas de calidad en la atención. Estas dificultades se presentan con mayor énfasis en los estados de más alta marginación y con mayor porcentaje de población indígena como Oaxaca, Guerrero y Chiapas..$^{20}$ En un foro organizado en 2010 por la Cámara de Diputados y Fundar, la representante de la organización Asesoría, Capacitación y Asistencia para la Salud de Chiapas, denunció que nueve de las diez clínicas obstétricas que existen en Chiapas carecen de medicamentos y equipos para la atención básica de la mujer embarazada. Este hallazgo muestra que a pesar del aumento en recursos para prevenir la mortalidad materna, no existe todavía ninguna garantía de que los recursos indispensables para lograr las metas del programa lleguen a su destino. ${ }^{21}$

Durante la contienda electoral de 2012 y en el inicio del periodo de gobierno del presidente priísta Enrique Peña Nieto (2012-2018), las propuestas de una "nueva" modificación a la protección en salud volvieron a ocupar un lugar importante en la agenda pública. Al igual que en otras oportunidades, los actores del sector se han manifestado desde dos posturas: quienes defienden el modelo de mercado regulado y plantean la posibilidad de corregir posibles fallas pero manteniendo el mismo esquema (Funsalud, 2010; Knaul y otros, 2012) y quienes proponen la necesidad de una reforma estructural que resuelva la fragmentación del sistema de salud y garantice el acceso para todos los ciudadanos a partir de criterios de equidad, de acceso efectivo y de la materialización del derecho a la salud garantizado por el Estado (Laurell, 2012a; Leal, 2012; grupo de académicos de la Universidad Autónoma Metropolitana, unidad Xochimilco (UAM-Xochimilco, 2013).

20 Estas reflexiones pueden ampliarse en el comunicado sobre la muerte materna en México divulgado por la Coalición por la Salud de las Mujeres el 9 de mayo de 2012 y en la página del Observatorio de la Mortalidad Materna en México.

21 Para ampliar esta información se puede consultar la nota de Ángeles Marínez en el periódico La Jornada del 30 de septiembre de 2010. 
El gobierno del presidente Peña Nieto ${ }^{22}$ ha manifestado su compromiso de continuar apoyando al SP, modernizar la infraestructura del sector y fortalecer las acciones de prevención de la salud. También ha planteado la necesidad de considerar la creación de un sistema único de salud que integre los esfuerzos de la SSA, de las instituciones de seguridad social y del sector privado. Este último proyecto se articula con la propuesta de Funsalud de realizar una reforma fiscal para conformar un fondo único mancomunado que permita financiar el sistema mediante impuestos generales. Se busca la creación de una instancia articulada, el establecimiento de un impuesto de consumo y un cambio en el sistema de contribuciones del IMSS y del ISSSTE. Este proyecto ha generado distintas reacciones por parte de los actores del sector.

La discusión de fondo se centra en aspectos como el logro de la cobertura universal, la definición de la protección en salud y las posibilidades de un sistema de salud como el mexicano para integrar los distintos recursos e intereses de los actores del sector. Este debate, en otras palabras, parece reciclar todas las iniciativas de los últimos treinta años, del intento fracasado de construir en 1983 un Sistema Nacional de Salud que integrara estructural y financieramente al sector, y no sólo de manera programática, y que posicionara a la SSA como cabeza del sector en su totalidad, y no como cabeza de sector de la población más pobre (Brachet-Márquez, 2010b).

Según los defensores del modelo, "el pluralismo debe seguir siendo el eje orientador, porque desde esta perspectiva puede lograrse una distribución de poder más equilibrada y evitar los extremos del monopolio en el sector público y la atomización en el sector privado" (Grupo de Trabajo Funsalud, 2013: 34). De esta manera, los actores clave en la consolidación del modelo de mercado regulado desde los años ochenta han vuelto a plantear la necesidad de reorganizar financieramente al sector y de consolidar la separación de funciones. Sin embargo, sobre este último tema han incorporado recientemente un elemento adicional: la creación de una instancia intermediadora (conformada por entidades públicas y privadas) para el manejo de los recursos y su canalización a las entidades prestadoras de servicios (Grupo de Trabajo Funsalud, 2013). Curiosamente, esta propuesta

22 Pese al apoyo de Peña Nieto al SP, no deja de ser interesante que el titular de este gobierno de la Secretaría de Salud, Gabriel O'Shea, cuestione los alcances de la promocionada cobertura universal. Recientemente ha manifestado que en la práctica este programa no es otra cosa que una empresa aseguradora, dado que se preocupa más por atender al mayor número de personas que por atender el desarrollo y el crecimiento de la infraestructura en salud en los estados (declaración publicada en el periódico El Universal el 5 de marzo de 2013. En: http://www. eluniversal.com.mx/notas/907743.html). 
deja de lado las evidencias tan valoradas en otros momentos por los reformadores como las que plantean expertos y consultores de salud como los profesores William Hsiao y Thomas Bossert, quienes han advertido que

estas instancias administradoras pueden volverse políticamente muy poderosas y frenar los esfuerzos para regularlas; los casos de Estados Unidos y Colombia son un buen ejemplo de esto. A partir de estas experiencias hemos recomendado que no se creen porque puede resultar un camino muy costoso que no necesariamente mejora la eficiencia del sistema de salud [...] una vez creadas estas empresas privadas, es imposible volver atrás, y es muy difícil garantizar una regulación estricta que las haga cumplir para mejorar la calidad y bajar los costos de los servicios (Hsiao y Bossert, 2009: 10-12).

Las diferencias entre las posturas de los actores del sector en estos proyectos de reforma se evidenciaron en el foro "Protección social en salud", realizado en la ciudad de México en abril de 2013 y convocado por la OPS y la Secretaría de Salud. En este foro participaron 60 representantes de diferentes instituciones y manifestaron sus puntos de vista sobre los proyectos de cambio para el sector. Los delegados del IMSS y del ISSSTE, por ejemplo, expresaron que no permitirían que la nivelación de los servicios se haga con el referente del SP, debido a que esto iría en detrimento de los beneficios ganados por los derechohabientes y del principio de solidaridad que rige. En sus palabras, la nivelación tendrá que hacerse "hacia arriba". También plantearon la necesidad de separar la provisión de servicios de salud de las pensiones para dar viabilidad a la posible mancomunación de los fondos. Entre las voces opositoras a la propuesta oficial que se escucharon en el mencionado foro, estuvieron las de un grupo de académicos de la UAM-Xochimilco, quienes han mantenido una postura crítica ante lo que denominan la implementación de un paradigma de mercado en la salud. Este grupo plantea que "el derecho a la salud tendría que ser el punto de partida para discutir sobre política de salud en condiciones democráticas [...] por tanto la prioridad debería ser la formación de un sistema único de salud de base pública e integral, solidario y equitativo, aspectos que anteceden la preocupación por los componentes financieros del sistema". Desde esta perspectiva, el IMSS podría ser la base para construir un Sistema Único de Salud (Relatoría Foro OPS, SSA, CIESS, 2013).

Aunque estas posturas parecerían mostrar que existe un acuerdo sobre la necesidad de avanzar hacia la universalización de la salud, difirieron en el camino para lograrla. Mientras la perspectiva dominante profundiza 
en las estrategias del "mercado regulado", la minoritaria ha argumentado sobre la necesidad de consolidar la centralidad del protagonismo estatal a partir del fortalecimiento de los sistemas públicos de salud. Entre los dos no se vislumbran posibles negociaciones.

\section{Reflexiones finales}

El recurrido presentado en este trabajo evidencia que la perspectiva políticaideológica para reformar el sistema de salud mexicano surgido en los años ochenta ha tenido continuidad. Se ha caracterizado por incorporar gradualmente al sector privado en la salud. Aunque los postulados del modelo conocido como "pluralismo estructurado" o "mercado regulado" no han logrado extenderse completamente a instituciones como el IMSS o el ISSSTE, siguen siendo el marco más importante para estructurar los proyectos de reforma presentados por el Ejecutivo para el sector salud (sobre todo desde el año 2000). Asimismo, los actores que impulsaron las reestructuraciones iniciales y que han ocupado posiciones clave en distintas instancias desde hace varios años (como Julio Frenk en la redacción de los informes del BM -1997 - , de la OPS - 2000 - , y como secretario de Salud entre 2000 y 2006), siguen teniendo un lugar definitivo en las discusiones y en las decisiones respecto al rumbo de las políticas de salud. Funsalud, organismo del sector privado, se ha fortalecido en estos años y convertido en uno de los actores con mayor peso en las decisiones del SPSS. Esta instancia pasó de ser asesora y copartícipe en el diseño de la reforma a convertirse en figura permanente en la definición de las orientaciones del SPSS. Sus análisis, basados en una perspectiva económica sobre la salud, se han vuelto un referente técnico sobre el que se suelen sustentar las evidencias de los cambios ocurridos como resultado de las reformas.

Los opositores a la visión oficial han sido generalmente marginados de las discusiones, y su insatisfacción con respecto a las decisiones se ha dejado ver al momento de la implementación del SP, cuando han salido a relucir las consecuencias de no incluir en las negociaciones a todos los actores del sector. Otros actores inconformes continúan llamando la atención sobre la necesidad de considerar los factores de desigualdad que permanecen en el sistema de salud debido a las diferencias en el acceso a los servicios o las posibilidades que este sistema de aseguramiento ofrece para hacer efectivo el derecho a la salud. Sin embargo, sus posturas siguen teniendo poco eco dentro de los proyectos de reforma, debido a que tampoco cuentan con los recursos institucionales y de poder (cargos en instancias decisorias, 
divulgación masiva de sus propuestas, apoyo del Ejecutivo) como lo han hecho los reformadores.

Aunque en sus discursos públicos los líderes priístas han mencionado la necesidad de universalizar la salud y de la inclusión con calidad, en la práctica estos actores legitimaron y apoyaron (y continúan apoyando) la introducción de la perspectiva que prioriza la estabilidad financiera. Por su parte, aunque el PRD ha sido consecuente en su oposición a este enfoque, su postura no ha tenido el peso suficiente para alterar las decisiones que atañen a la salud. La dinámica para incorporar los cambios - especialmente en el subsector público de la salud - muestra nuevamente que la salud es algo más que un asunto técnico y por sí mismo constituye una arena política en la que se juegan diversos recursos e intereses. Además, deja ver que las negociaciones en el ámbito parlamentario no son suficientes para lograr las condiciones para su puesta en marcha.

La discusión sobre la necesidad de una "nueva" reforma para universalizar la salud ha vuelto a tomar un lugar importante en la agenda del recién electo gobierno de Peña Nieto, sin embargo, aún no se ve muy claro el futuro del SP, no sólo por la sustentabilidad financiera que el programa requiere, sino porque cada vez son más los actores que exigen no sólo la universalización del aseguramiento sino también, y sobre todo, del acceso con calidad en igualdad de condiciones (contributivo o no contributivo), además de una respuesta adecuada a las demandas actuales surgidas de un cuadro epidemiológico complejo en el que predominan las enfermedades crónico-degenerativas no transmisibles (Uribe, 2011). Aunque la protección financiera constituye un pilar fundamental dentro de cualquier sistema de salud, no es un elemento suficiente si no incluye mecanismos adecuados de equidad, solidaridad y garantía de acceso a los servicios independientemente de la capacidad de pago de las personas (CEPAL, 2006; OIT, 2007).

En resumen, todavía parece estar lejos el consenso entre quienes siguen defendiendo la ruta del pluralismo estructurado como el mejor camino para reformar el sector salud mexicano y quienes aducen que es necesario introducir una perspectiva fundamentada en un tipo de ciudadanía basada en derechos exigibles. 


\section{Bibliografía}

Abrantes Pêgo, Raquel, 2010, Salubristas y neosalubristas en la reforma del Estado: grupos de interés en México e instituciones públicas de salud, 1982-2000, México, El Colegio de Michoacán.

Abrantes Pêgo, Raquel, 2006, “La reforma del IMSS, el poder de decisión del Presidente y del corporativismo en el proceso de decisión en el contexto de democratización", en V Congreso Nacional Tamet 2006.

Almeida, Celia, 2005, "Reforma del sector salud y equidad en América Latina y el Caribe: conceptos, agenda, modelos y algunos resultados de implementación", Gerencia y Políticas de Salud, vol. 4, núm. 9, Colombia, pp. 6-60.

Banco Mundial (BM), 2010, “México: respalda BM con 1,250 millones al Seguro Popular", comunicado de prensa 010/315/LAC. Disponible en: http://web.worldbank.org/WBSITE/EXTERNAL/BANCOMUNDIAL/NEWSSPANISH> consultada en abril de 2012.

Blanco Gil, José y López Arrellano, Oliva, 2007, “Las vertientes privatizadoras del seguro popular de salud en México", Salud Problema, núm. 1-2, año 1, enerojunio y julio diciembre, pág. 55-62.

Boltvinik, Julio,(199), "Privatizar para segregar", La Jornada, viernes 3 de abril de 1998, <http://www.jornada.unam.mx/1998/04/03/bolvitnik.html>.

Brachet-Márquez, Viviane (2010), “Seguridad social y desigualdad, 1910-2010”, en Fernando Cortés y Orlandina de Oliveira (eds.), Los grandes problemas de México: desigualdad social, México, El Colegio de México, pp. 181-210.

Brachet-Márquez, Viviane (2010b), “Salud y seguridad social en México: 1919-2008 ¿Quién decide?”, en José Luis Méndez (ed.), Del Estado autoritario al gobierno dividido; situación y perspectivas del Estado y las políticas públicas en el régimen democrático presidencial mexicano, México, El Colegio de México, 2010.

Brachet-Márquez, Viviane (2007), “Las reformas de los sistemas de salud y previsión social en México 1982-2000”, en Viviane Brachet-Márquez (ed.), Salud pública y regímenes de pensiones en la era neoliberal: Argentina, Brasil, Chile y México 19802000, México, El Colegio de México, pp. 291-348.

Comisión Económica para América Latina y el Caribe (CEPAL), 2006, La protección social de cara al futuro: acceso, financiamiento y solidaridad, Santiago de Chile, CEPAL. 
Durán, Luis, Vanesa Rodríguez, Carmen Hernández y Luis A. García, 2010, “Los seguros médicos privados en México: su contribución al sistema de salud", Resumen Ejecutivo de Investigación, Medicina y Salud, UNAM, pp. 1-25.

Enthoven, Alain C., 1980, Health Plan: the Only Practical Solution to the Soaring Cost of Medicale Care, Reading, M., Addison-Wesley.

Frenk, Julio, 2012, "La salud como derecho ciudadano", Nexos, julio. Disponible en: <URL:http/ / nexos.com.mx/?P=leerarticulo\&Article=2102556>, consultada en mayo de 2012.

Frenk, Julio, 2005, "El seguro popular de salud es un instrumento de justicia social", Seguro Popular de Salud: siete perspectivas-Salud Pública de México, vol. 46, núm. 6, México, pp. 588.

Fundación Mexicana para la Salud (Funsalud), 2006, La salud en México 2006-2012: visión de Funsalud, México, Funsalud.

Funsalud, Economía y Salud, 1994, Propuesta para el avance del sistema de salud en México, México, Funsalud.

Gómez, Thelma, 2010, "Seguro Popular, derroche sin resultados", El Universal, lunes 12 de abril.

Gómez Dantés, Octavio, 2005, "Seguro popular: siete perspectivas", Salud Pública de México, vol. 46, núm. 6, nov.-dic. 2004, México, pp. 585-588.

González Rossetti, Alejandro y Olivia Mogollón, 2002, “La reforma de salud y su componente político: un análisis de factibilidad", Gaceta Sanitaria, vol. 16, núm. 1, México, pp. 39-47.

González, Jorge I., 2001, “Imparcialidad financiera y equidad: otra lectura del informe de la Organización Mundial de la Salud", año 2000, Gerencia y Políticas de Salud, núm. 1, pp. 39-47.

González, Luz M., Gustavo Nigenda, María González y Michel Reich (2011), “Separación de funciones en el Sistema de Protección Social en México: avances y retos", Salud Pública de México, vol. 53, suplemento 1.

Grupo de Trabajo de la Fundación Mexicana para la Salud (Funsalud), 2013, “Universalidad de los servicios de salud en México", Salud Pública de México, vol. 55, número especial. 
Hernández, Mario, 2008, “El concepto de equidad y el debate sobre lo justo en salud", Salud Pública, núm. 10, pp. 72-82.

Hernández, Mario, 2003, “El enfoque sociopolítico para el análisis de las reformas sanitarias en América Latina", Revista Cubana de Salud Pública, vol. 29, núm. 3, Cuba, pp. 228-235.

Holzmann, Robert y Steen Jorgensen, 2000, “Manejo del riesgo social: un nuevo marco conceptual para la protección social y más allá", Serie de discusión de documentos sobre la protección social, núm. 0006, Washington, Banco Mundial, Unidad de Protección Social, pp. 73-106.

Homedes, Nuria y Antonio Ugalde (eds.), 2006, Descentralizing Health Services in Mexico: A Case Study in State Reform, Center for US-Mexican Studies, University of California, $314 \mathrm{pp}$.

Homedes, Nuria y Antonio Ugalde, 2011, “Descentralización de los servicios de salud: estudios de caso de seis estados mexicanos", Salud Pública de México, vol. 53, núm. 6, Dic.

Instituto Mexicano del Seguro Social (IMSS), 1996, Ley del Seguro Social, México, IMSS.

Instituto Mexicano del Seguro Social, 1995, Diagnóstico, México, IMSS.

Instituto Nacional de Estadística y Geografía (INEGI), Comisión Nacional de Derechos Humanos (CNDH), 2011, "Informe sobre el derecho a la salud en México", INEGI, CNDH, Naciones Unidas, México, p. 67.

Infante, Alberto, Isabel Mata y Daniel López, 2000, “Reforma de los sistemas de salud en América Latina y el Caribe: situación y tendencias", Revista Panamericana de Salud Pública, vol. 8, núms. 1-2, OPS, pp. 13-20.

Knaul, Felicia, Eduardo Gonzales-Pier, Octavio Gómez-Dantés et al., 2012, "The Quest for Universal Health Coverage: Achieving Social Protection for all Mexico", Health Policy, Published online August 12.

Lakin, Jason M., 2010, “The End of Insurance?, Mexico's Seguro Popular 2001-2007”, Journal of Health Politics, Policy and Law, vol. 35, núm. 10, June.

Laurell, Asa Cristina, 2012a , “Ganadores y perdedores de la seguridad social universal”, Columna de opinión-La Jornada, México, miércoles 9 de mayo.

Laurell, Asa Cristina, 2012b, “La cobertura médica del seguro popular", Columna de opinión-La Jornada, México, miércoles 25 de enero. 
Laurell, Asa Cristina, 2010, “La segunda reforma de salud: aseguramiento y compraventa de servicios", Salud Colectiva, vol. 6, núm. 2, agosto, México, pp. 137-148.

Laurell, Asa Cristina, 2009, "El seguro popular: mitos y realidades", La Jornada, México, febrero 19, consultado en mayo de 2012.

Laurell, Asa Cristina, 2005, "El programa de servicios médicos y medicamentos gratuitos más apegado a la esencia del derecho a la salud que el Seguro Popular", Salud Pública de México, INSP, vol. 47, núm. 1, enero-febrero, México, pp. 82-86.

Lavielle, Griselda, 2012, "Resultados negativos del Seguro Popular en los Estados”, en Fundar Pesos y Contrapesos, Informe de Fundar, mayo, México. Disponible en: <http:/ / fundar.org.mx/pesosycontrapesos/?p=94, consultada en mayo de 2012>.

Leal, Gustavo, 2012, AMLO: ¿modificar o sustituir el Seguro Popular?", en La Jornada, México, junio. Disponible en: <http://www.jornada.unam.mx/2012/06/09/ politica/022a1pol>.

Londoño, Juan Luis y Julio Frenk, 1997, Pluralismo estructurado: hacia un modelo innovador para la reforma de los sistemas de salud en América Latina, Washington, Serie de documentos de trabajo; 353.

Navarro, Vicente (2001), "Evaluación del informe sobre la salud en el mundo, 2000", Gerencia y Políticas de Salud, núm. 1, noviembre, pp. 30-38.

Nigenda, Gustavo, 2005, “El Seguro Popular de salud en México: desarrollo y retos para el futuro", Banco Interamericano de Desarrollo, núm. 2, Nota Técnica sobre Salud, consultado en mayo de 2011.

Organización Internacional del Trabajo (OIT), 2007, “Protección Social de la Salud: una estrategia de la OIT para el acceso universal a la asistencia médica", en Documento de consulta OIT. Disponible en: <http://www.ilo.org/public/spanish/protection/secsoc/downloads/healthpolicy_sp.pdf>, consultada en agosto de 2012.

Organización Panamericana de la Salud (OPS), 2002, “Ampliación de la Protección Social en Materia de Salud: iniciativa conjunta de la Organización Panamericana de la Salud y la Organización Internacional del Trabajo", en 26a. Conferencia Sanitaria Panamericana, 54a. Sesión del Comité Regional, Washington.

Ortiz, Mauricio, 2006, El seguro popular: una crónica de la democracia mexicana, México, Secretaría de Salud/Fundación Mexicana para la Salud Fondo de Cultura Económica. 
Pérez, Mariana, 2010, “¿Avances para la realización del derecho a la salud en México?", en Mariana Pérez (ed.), Cinco miradas sobre el derecho a la salud: estudios de caso en México, El Salvador y Nicaragua, México, Fundar, pp. 117-176.

Perticara, Marcela, 2008, "Incidencia de los gastos de bolsillo en salud en siete países latinoamericanos", en Serie de Políticas Sociales, núm. 141, Santiago de Chile, CEPAL, pp. 1-68.

Sáenz, Miguel, 1996, La reforma a la seguridad social mexicana, cambios a la iniciativa presidencial, México, El Cotidiano.

Sistema de Protección Social en Salud (SPSS), 2012, Catálogo Universal de Servicios de Salud-Causes, México, Comisión Nacional de Protección en Salud/Seguro Popular.

Sistema de Protección Social en Salud (SPSS), 2011, Informe de Resultados 2011, México, Gobierno Federal Seguro Popular.

Soberón, Guillermo, 2001, "La participación del sector privado", en Gaceta Médica de México, vol. 137, núm. 5, sept.-oct., México, pp. 437-43.

Tamez, Silvia y Catalina Eibenschutz, 2008, “El Seguro Popular de Salud en México: pieza clave de la inequidad en salud", en Salud Pública, vol. 10, núm. 8, diciembre, México, pp. 133-145.

Tamez, Silvia, Claudia Bodek y Catalina Eibenschutz, 1995, “Lo público y lo privado, las aseguradoras y la atención médica en México", en Cuadernos de Salud Pública, vol. 11, núm. 4, México, pp. 139-153.

Ulloa, Odilia, 1996, Nueva Ley del Seguro Social: la reforma previsional de fin de siglo, México, El Cotidiano.

Uribe Gómez, Mónica, 2011, “¿Mercado regulado o competencia sin control? Las reformas de la salud en Colombia, 1995 2011", en Los vaivenes de las políticas sociales en Argentina, Colombia, Chile, México y Uruguay: ¿neo o posneoliberalismo?, México, Porrúa, pp. 33-80.

Valencia, Enrique, David Rodríguez y Darcy Tetreault, 2011, “Sistema de Protección Social en México a inicios del siglo XXI", documento proyecto Asistencia social: reducción de la pobreza y redistribución del ingreso a través del programa de transferencias condicionadas, México, CEPAL-ASDI.

Recibido el 9 de noviembre de 2012 Aceptado el 29 de abril de 2013 